\title{
The physiological and biomechanical process of motor retraining for movement dysfunction
}

\begin{abstract}
The aim of this work is to consider how normal and abnormal processes of biomechanics occur in relation to neuronal movement processes within the primary motor cortex (PMC); and also to consider how kinetic therapy can potentially be used to develop movement retraining strategies within neuronal movement to further biomechanical function processes. The work will also be looking at how the understanding of a pathologica condition and its effects on normal biomechanics from a motor control perspective are essential for considering movement retraining strategies for the clinician, whilst also taking into account the importance of patient education for the increased understanding of normal and abnormal movement processes in effective symptom management. For the purpose of clinical illustration, this work will be considering lumbar disk herniation and its direct effect on neuronal transmission in sciatica and other nerve impingement conditions. This will be discussed in relation to the potential alteration of neuronal imprinting within the PMC. Neuronal imprinting and its relevance to biomechanical movement processes will be discussed in relation to general functionality. Kinetic retraining strategies will be examined in this work, considering whether-by retraining normal biomechanical movement patternskinetic therapy can strengthen cortical movement representations within the PMC to affect actual biomechanical movement processes.
\end{abstract}

Keywords: movement disorder, kinetic therapy, biomechanics, neuronal imprinting

\author{
Volume I Issue 4 - 2015
}

\author{
William James Cobb, Edwin Purcell, Rebecca \\ Louise Cusack \\ Department of Physiology, University of Medicine and Health \\ Sciences (UMHS), USA
}

Correspondence: William James Cobb, Department of Physiology, University of Medicine and Health Sciences (UMHS), USA,Tel 01235833 I73,Email wcobb@umhs-sk.net

\author{
Received: November 0I, 2015 | Published: November 20, \\ 2015
}

\section{Introduction}

Cordo and Harnad, ${ }^{1} \mathrm{Key}^{2}$ and Shaw et al. ${ }^{3}$ describe imprinting as the processing control point of functionality within a movement or a process of movements. The imprinted movement, as stored in the cortical space, is a specific movement, and therefore unique with regard to where the movement occurs in/on the body. The movement process is not only dependent on the PMC and cortical space, but also on the many complex neural pathways that transmit the desired pattern of movement to the desired location on the body. ${ }^{4}$ During the processes of normal functional movement control or indeed abnormal movement control, a biological/biochemical chain of events takes place that allows for movement and hence functionality to occur. Whether movement is normal or abnormal, the process of functionality has a beginning and an end point. The beginning of a given movement process starts in the PMC, as stored in cortical space, and the end point is the actual completed biomechanical movement process. An example of this is to imagine the flexion of the finger. From the point in which the desired movement is processed within the PMC where the movement is imprinted within cortical space, to the point in which it is transmitted to the desired location (flexion of the fingers), the signal has completed a circuit that has resulted in a functional biomechanical movement process occurring. ${ }^{5}$ Movement itself, and hence functionality, is variant dependent on the level of complexity of the desired movement pattern. Finer movements such as those controlling the hands and feet require more precision, and in turn, more cortical space within the motor cortex in order to achieve increased complexity within biomechanics. In opposition to this, broader and less refined movements - such as flexion of the lower limb-require less cortical space as the actual movement process itself is less biomechanically complicated and therefore requires less processing power and thus less cortical space. ${ }^{6}$ The reasons why abnormal movements occur can be extremely diverse. These movements can be caused by underlying pathological changes such as neural degenerative diseases; , trauma relating to cortical damage; carcinoma; and indeed reduced movement pattern recognition within the PMC. Movement pattern recognition can be the point in which a movement is no longer holding a recognizable, biomechanically functional form within the PMC, thus resulting in a movement that could be described as altered or abnormal. ${ }^{7}$ Whether the actual movement process as represented in the PMC is functionally formed or not, the movement may still be abnormal when actually transmitted as a formed movement, as the area stimulated may have degraded, and altered neural path(s) or biomechanical alteration to the limb may have occurred. In trauma this in turn can change normal biomechanical movement, thus altering function. ${ }^{8}$

Whatever the underlying pathological change may be, the agreed conclusion is that when a movement is abnormal, the underlying blueprint of the movement within the PMC is affected-or the actual area/location of movement is affected. Whether the movement has been affected due to the underlying blueprint being damaged, or indeed the neural paths transmitting the movement pattern themselves being damaged, the eventual pattern of movement itself, if left untreated, may result in the PMC becoming corrupted, thus potentially altering functional movement processes. ${ }^{9}$ Loss of functional movement control that has a neuronal cause is essentially a movement pattern recognition fault that has resulted in variant/abnormal movement(s) that potentially may cause gross biomechanical instability. ${ }^{10}$ Biomechanical stability is as dependent on the PMC as the PMC is dependent on the biomechanical stability of the functional area being activated. ${ }^{11}$ Anatomically, the PMC is located in the frontal lobe of the brain by the precentral gyrus (PG). The PMC generates neural impulses, which control the implementation of movement processes and signals that cross the body's midline, eventually activating skeletal muscles on the opposite side. This opposition in effect is due to each 
hemisphere of the brain controlling the opposite side of the body. ${ }^{12,13}$ An example of how this opposition effect works would be to imagine the flexion of the left leg. This action of flexion has been initiated at the right hemisphere of the brain within the PMC, using a smaller amount of cortical space due to the movement being broader and less refined. If the right leg were to be flexed, then the left hemisphere would be responsible for the actual movement process. This understanding of counteracting opposition is important for both the clinician and the patient alike, as knowing where a movement problem/dysfunction may be occurring may in turn guide the course of movement retraining strategies. ${ }^{14}$ Functionally, and therefore biomechanically, areas of the human body are represented in the PMC and these areas are arranged somatotopically. ${ }^{15}$ Somatotopically interpretation, especially in relation to movement processes, can be better understood by using the homunculus as a map of somatotopically interpretation. When looking at the homunculus, the foot can be seen next to the leg, which is then next to the trunk, which in turn is next to the arm and the hand - and so on. The brain's specific areas of interpretation as represented on the homunculus are very important clinically, as they allow for the clinician to understand where the origins of the movement(s) may be occurring (Diagram 1). ${ }^{16}$

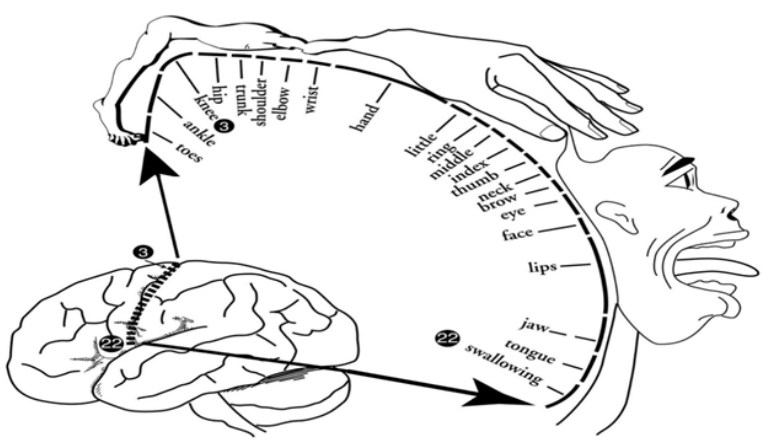

Diagram I Somatotopical interpretation on the homunculus model of functionality. ${ }^{16}$

The understanding of movement and the processing areas of the brain that are involved in order to perform these are essential when considering abnormal movement patterns. Through the understanding of movement processing areas of the brain and their relation to the actual physical movement process, both the clinician and the patient alike can develop effective movement retraining strategies built on physiological understanding. ${ }^{17}$ The clinician who educates the patient is more likely to build rapport and trust, and therefore may then involve the patient in treatment/therapy options. Bosworth ${ }^{18}$ suggests that the involvement of the patient in treatment/therapy options not only helps build rapport, but may also help guide the clinician's strategies for patient education. Bosworth ${ }^{18}$ suggested that where the clinician adopts the approach of educating the patient on a condition and hence a treatment, this may result in the patient wanting to engage in active therapy/treatment, and in turn, in therapy/treatment design. When considering movement control and the retraining of abnormal movement, it is important to consider the affected area and the complexity of biomechanical mechanisms within that area. Certain areas of the human body require finer movement control than others. Fine movement control, as seen in the fine and precise movements of the fingers and toes, takes more processing power and hence requires more cortical space. ${ }^{6}$ The actual process of movement control, whether it be refined-or less refined as in flexion of the upper leg-requires not only the source of movement as represented in the PMC, but also the ability of the movement to be biomechanically supportable within the desired area of movement. ${ }^{19}$
An example of fine and indeed broad motor control within functionality, and how these points could be considered in movement retraining is that of the flexion of the fingers, referred to at the beginning of this work. When considering disc herniation and its potential direct effect on the sciatic nerve or indeed on nerve impingement in general, the patient who suffered a disk herniation resulting in compression of the lumbosacral plexus may experience a number of movement disorders or clinical manifestations. For instance, if the patient had reduced movement of the foot and indeed the entire leg, then the broad and fine movement representations within the PMC may be altered/ corrupted thus altering function. This could also be the other way around if the limb is affected and unable to biomechanically support the desired movement process as represented in the PMC. From a $\mathrm{PMC}$ to functional movement process perspective, the alteration could be due to the fact that the impingement is no longer allowing for nerve impulses to stimulate biomechanical action within the given limb. Biochemically speaking, this could have been the initial instigator, followed by the PMC essentially forgetting the desired movement pattern. When considering kinetic retraining strategies, this could be important, as even if the impingement is corrected, the movement as represented in the PMC may be corrupted, essentially having a distorted stored movement pattern resulting in an uncontrolled movement. ${ }^{11}$ The clinician with the underpinning knowledge of the physiological and biomechanical processes of movement could potentially consider not only the biomechanical alteration of movement itself, but also the neural beginnings of functionality. The clinician can consider retraining strategies based both on the biomechanical features and the neural features, therefore considering the beginning and ending points of functionality. ${ }^{4}$ For the purpose of clinical illustration, let us imagine that a patient has had an L5 disc herniation which has resulted in the inability to dorsiflex the foot. The anatomical structures of the foot are complex, as biomechanically they have to perform complex and intricate movement patterns. All of these fine movements within the foot require a substantial number of complex neural processes that in turn require increased cortical space within the PMC. ${ }^{20}$ The clinical presentation of the patient who has had problems with the ability to dorsiflex the foot (abnormal movement) may suggest that the flexors have lost the impulse of flexion as represented in the PMC. The clinician who is considering movement retraining strategies may need to consider the following points in relation to this movement fault(s):

i. Is the flexor(s), in neurostimulatative terms, underactive and are the extensors now overactive?

ii. Could overcompensation of the stimulus to the extensors be resulting in over-action of the extensor muscle groups?

iii. Could the flexor muscle group be becoming overstretched, resulting in the muscles being biomechanically unstable in opposition to the extensor group(s) and could this be causing co-contraction?

iv. Would retraining of the extensor muscle(s) allow for more stability in opposition to the force exerted by the opposing flexors?

v. Would retraining the muscle group(s) allow for divergent neural paths to form allowing for a functional biomechanically stable movement pattern to form within the PMC?

The above questions are far from all-encompassing and only explore some of the many potential reasons behind a movement fault(s). The clinician, who is diagnostically considering the above points, could also consider whether the nerve is damaged either by trauma or pathologically, as reflected in this scenario. Nerve damage 
can not only cause the loss or reduction of function, but can also cause circulatory problems to the area, which in turn may cause unhealing wounds and muscle atrophy-all of which can lead to increased biomechanical instability. ${ }^{21}$ Additionally, the patient who has had a lumbar disc herniation may present with symptoms in the affected limb and in the gluteal region, which can be bilateral or unilateral dependent on the degree of nerve impingement such as pain, burning sensations, tingling sensations, weakness, numbness and lack of motor control. ${ }^{22}$ As mentioned in the scenario, the ability to dorsiflex the foot, which is required when making a stepping motion, may be compromised in the patient with sciatica. This is an important clinical point, as step tests could be performed to look for abnormal movement patterns in walking, climbing, sitting to standing etc. The clinician with the underlying understanding of the physiological processes relating to biomechanical movement should also be able to sufficiently assess for sensory input via tests such as the pin prick and pressure sensation test. ${ }^{23}$ If the afferent fibres of the nerve are affected the sensory areas may be affected, resulting in paresthesia, which clinically manifests as tingling or pins and needles in the affected limb peripherals. In opposition to this, if the afferent fibres are over stimulated a hypoesthesia may result which has been described as causing over sensation of receptors resulting in increased sensation to touch, temperature, and pain. ${ }^{24,25}$

Wong and Transfeldt ${ }^{26}$ and Acton $^{27}$ describe classic clinical manifestations of sciatica in relation to pain when the patient adopts the sitting or squatting position. Both also describe patients reporting pain in the gluteal region whilst adopting a sitting position, which can be accompanied by pain in the leg classically described as a burning or tingling sensation that emanates down the leg. This in turn can also be accompanied by weakness, numbness, or difficulty moving the leg or foot. Dependent on what nerve or group of nerves are being impinged, sciatica can affect one side of the lower body or both (bilateral impingement). Progressive lower extremity weakness and/or loss of bladder or bowel control can suggest that the nerve damage is affecting $\mathrm{S} 2,3,4$ at which point incontinence may well become a clinical feature. ${ }^{28}$ Anatomically, the sciatic nerve originates from the distal spinal cord and extends almost the entirety of the hind limb and is a combination of nerves from L4-S4. The sciatic nerve innervates the majority of the hind limb and is a mixed function nerve made up of axons of sensory and motor neurons. As the sciatic nerve progresses distally it gives off branches along the hind limb; these branches contain motor and sensory neurons that are involved in the control of the muscle groups of the upper leg. The flexor and extensor groups of the nerve include the hamstring group, which flexes the upper leg and the lower leg and contains both flexors and extensors. ${ }^{28}$ The sciatic nerve is comprised of the axons of hundreds of neurons. The axons themselves vary in diameter ranging from $<1$ to $20 \mathrm{~mm}$. This is clinically significant, as conduction velocity is proportional to axon diameter, and therefore neural signal speed and strength is variable dependent upon this. Within the sciatic nerve itself, the conduction velocity of the nerve has been measured and shown to vary from 0.2 to $150 \mathrm{~m}$ sec- $1 .^{29,30}$ Again, this is also very clinically important when considering the reflex arc within the point of a stimulus to the point of action for biomechanical functional movement tests. Neurons themselves are categorized according to morphology and/or function (sensory or motor) and have been classified into groups such as type A fibres, type B fibres and type $\mathrm{C}$ fibres.

Type A fibres are described as having a larger axon diameter with thicker myelin sheaths and in turn have an increased conduction potential which ranges from $30-150 \mathrm{~m}$ sec-1. ${ }^{29,30}$ Type A fibres are mainly motor efferent neurons and are responsible for the control of skeletal muscles. The sensory (afferent) neurons express information from receptors in the muscles, joints, and epidermal tissues to the spinal cord. ${ }^{29,30}$ Type B fibres are described as having less welldeveloped myelin sheaths and conduction velocities that range from about $3-15 \mathrm{~m} \mathrm{sec}-1 .{ }^{29,30}$ The majority of the fibres form part of the autonomic nervous system's efferent pathways. These efferent pathways are responsible for the innervation of internal organs and blood vessels whilst regulating activity. ${ }^{29,30}$ Type $\mathrm{C}$ fibres have the smallest fibres in diameter and lack myelin sheaths, thus, in turn, lack the potential for increased levels of conduction. This means that the type $\mathrm{C}$ fibres have low conduction velocities of around $(<$ $2 \mathrm{~m} \mathrm{sec}-1){ }^{29,30}$ The majority of the fibres are efferent neurons of the sympathetic nervous system and afferent pain neurons..$^{29,30}$ Clinically, this is important, as understanding the groups of fibres allows for the clinician-and indeed the patient-to understand conduction in relation to biomechanical function as well as conduction in relation to pain and indeed to pathological change (Figure 1) (Figure 2). The understanding of the biomechanics of movement in considering the many intrinsic processes that takes place within overall functionality, allows the clinician and the patient alike to consider the signs and symptoms of the presenting condition(s) and in turn to plan appropriate treatment/therapy based on the best evidence base around the clinical manifestation. ${ }^{31}$ The greater the clinical/physiological understanding of the afferent and efferent processes in relation to functionality and control of functionality the broader the basis of treatment etiology. ${ }^{32}$ This incorporation of knowledge, and indeed information-sharing with the patient, may allow for broader understanding of other pathological changes that may be occurring as a result of efferent impulse changes in relation to the overall biomechanical changes. As discussed earlier within this work, the clinician with the understanding of the mechanisms of impulse convergence in relation to biomechanical movement function will be adept at recognizing other subtle changes that may be manifesting, such as decreased vascularity, increased or decreased sensation etc. ${ }^{33}$

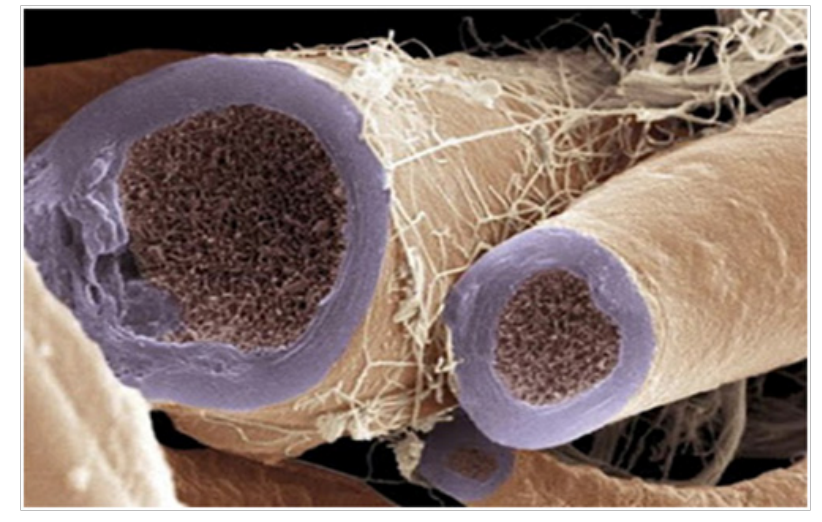

Figure I Nerve bundle. Colored scanning electron micrograph (SEM). ${ }^{29}$

Fundamentally, the understanding of a condition and the physiological processes within biomechanics and biomechanical movement help guide the clinician's reasoning in clinical decision making. ${ }^{34}$ A movement dysfunction/disorder may be due to a catalogue of reasons. Whatever the reasons behind a movement disorder, the fundamental changes within the physiological and biomechanical processes can be seen and at least to some degree understood, by the clinician with a background understanding in the subject area. ${ }^{35}$ As discussed in the body of this work, the clinician who not only educates him or herself on the physiological processes of movement but also on related issues, may be better equipped at conveying movement retraining strategies to patients through the 
process of clinician to patient education. ${ }^{36}$ This in turn allows for the clinician and indeed the patient to explore treatment options based around a strong underpinning knowledge of the subject area. The understanding of treatment options is very important, not only in building up trust between the patient and the clinician, but also for the appropriate treatment plans/strategies to be wholly adopted by the patient. ${ }^{37}$ The patient who understands his or her own condition(s) may be more adept at recognizing signs of improvement and indeed deterioration. This is extremely helpful for the clinician, as the patient who understands why these changes are occurring may be able to alert the clinician to change, thus allowing time for alternative strategies to be employed if needed. ${ }^{17}$ Patient education, and the point at which the patient and the clinician form a working bond, are areas of medicine and healthcare that have been extensively discussed. Several authors such as Lorig ${ }^{38}$ and Redman ${ }^{39}$ suggest that the clinician who educates his or her patients increases the chances of successful treatment implementation. This is an interesting point, as the patient who feels empowered by knowledge may well be more likely to engage with treatment options and in turn more likely to want to engage with treatment/therapy over time.

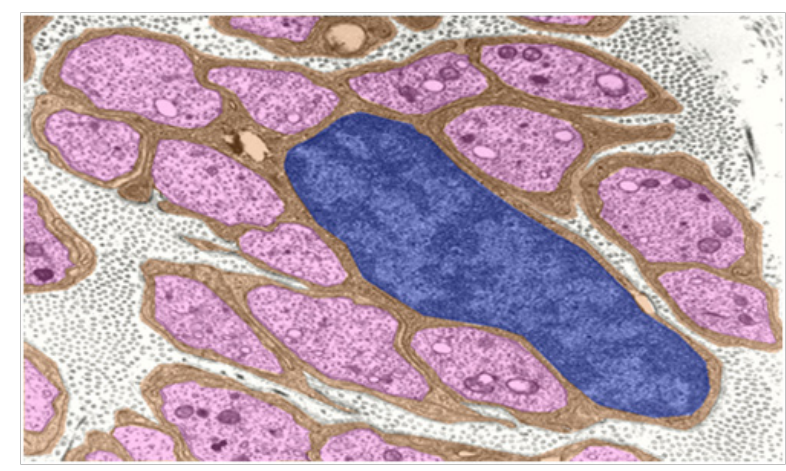

Figure 2 Unmyelinated nerve fibers do not have the concentric myelin sheaths that surround other types of nerve; instead the axons are enveloped within invaginations of Schwann cells cytoplasm. ${ }^{29}$

\section{Summary}

In summary, the clinician who has a broad understanding of the physiological and biomechanical processes of functionality has the ability to consider retraining of movement in a broader context The patient presenting with an abnormal movement may have a simple biomechanical fault which may be obvious and indeed may be treatable by opposition muscle retraining. This, however, is only part of the overall picture of function. The underlying neuronal link may also be affected, perhaps as a result of the biomechanical fault affecting imprinting or vice-versa. ${ }^{1}$ The clinician who considers both the beginning and the end of functionality looks at the overall picture of movement, and from this allows him or herself to consider strategies for retraining of abnormal movement patterns. ${ }^{31}$ The clinician who adopts the idea of linking functionality to both biomechanical features and indeed neuronal features will have a larger array of movement retraining techniques available. Abnormal movement related to retraining strategies is a very broad subject, which indeed requires further investigation into neuronal retraining. However, this work has considered some of the elements of functionality in relation to neuronal control of function looking at the link of biomechanics.

\section{Conclusion}

For development of personal future clinical practice related to some of the material covered in this work, it may be that by considering movement from a much broader perspective, better diagnostic skills
- and hence better treatment/retraining options-become available. The author of this work feels that, for his own clinical practice, rather than just considering movement and movement faults at the point of the biomechanical feature, by considering the processing point of function, further ideas on movement and movement retraining can be adopted. The author of this work also feels that by having an increased knowledge base on the neural links between function and dysfunction, the clinician may be able to efficiently diagnose aspects of neural change through diagnostic investigation of the movement fault. This is clinically very important, as the clinician with the ability to grasp the concepts of function from the neural to biomechanical point may be better equipped at recognizing not only deterioration in biomechanical movement processes, but also the deterioration in neural control as seen in neurodegenerative diseases. This could in turn be an alert to signs of such diseases, and potentially to the referral of the patient to appropriate healthcare professionals. The author feels that this work has raised some points surrounding the education of the patient in the context of understanding a condition from a physiological/biomechanical perspective. It may be that the clinicians who has the underlying broader knowledge of movement, function, and how retraining can be incorporated into this, will also have the ability to convey this to the patient. Can the link between knowledge and empowerment of the patient be bridged through broader understanding? Could this lead to more effective treatment strategies and indeed to success in treatment outcomes? These are some of the further questions that have been postulated by the author during this work in relation to clinician to patient education. Overall, the author feels that the broader the understanding of functionality, the broader the teaching and in turn retraining potential.

\section{Acknowledgements}

None.

\section{Conflict of interest}

Author declares that there is no conflict of interest.

\section{References}

1. Karen T Tylor. The Human Face. In: Tylor and Francis group, editor. Forensic Art and Illustration. New York: CRC Press; 2001:48-50.

2. Cordo P, Harnad S. Movement Control. UK: Cambridge University Press; 1994.

3. Key J. Back pain-A movement problem: a clinical approach incorporating relevant research and practice. London: Churchill Livingstone Elsevier; 2010.

4. Shaw GL, McGaugh JL, Rose SPR. Neurobiology of learning and memory. UK: Library of Congress Publishing; 1990.

5. Stirling J. Cortical functions. London and New York: Taylor \& Francis Publishing; 2002.

6. Bartlett R. Introduction to sports biomechanics. Abingdon, UK: Taylor and Francis Publishing; 2002.

7. Freberg AL. Discovering biological psychology. Washington DC: Library of Congress Publishing; 2009.

8. Schamberger W. The Malalignment Syndrome, diagnosis and treatment of common pelvic and back pain. Philadelphia: Churchill Livingstone Elsevier; 2012.

9. Lynn DJ, Newton HB, Grant AD. The 5-minute neurology consult. Philadelphia: Lippincott Williams and Wilkins; 2004.

10. Winter DA. Biomechanics and motor control of human movement. New Jersey: Wiley and Sons; 2009. 
11. Jankovic J. Parkinson's disease: clinical features and diagnosis. J Neurol Neurosurg Psychiatry. 2007;79(3):368-376.

12. Riehle A, Vaadia E. Motor cortex in voluntary movements: a distributed system for distributed functions. Washington DC: CRC Press, Taylor \& Francis Group; 2004.

13. Porth C. Essentials of pathophysiology: concepts of altered health states. 3rd ed. Philadelphia: Lippincott Williams \& Wilkins; USA: 2011.

14. Wassermann EM, Epstein CM, Ziemann U, et al. Oxford handbook of transcranial stimulation. Oxford: Oxford University Press; 2008.

15. Kandel ER, Schwartz JH, Jessell TM, et al. Principles of neural science. 5th ed. New York: McGraw Hill Publishing; 2013.

16. Tresilian J. Sensorimotor control and learning: an introduction to the behavioural neuroscience of action. UK: Palgrave Macmillan; 2012.

17. Bullock J, Boyle J, Wang MI. NMS Physiology. 4th ed. Philadelphia: Lippincott Williams and Wilkins; 2011.

18. Falvo D. Effective patient education: a guide to increased adherence. Burlington: Jones \& Bartlett; 2010.

19. Bosworth H. Improving patient treatment adherence: a clinician's guide. London: Springer Publishing; 2010.

20. Rhoades R, Bell DR. Medical physiology: principles for clinical medicine. 4th ed. Philadelphia: Lippincott Williams \& Wilkins; 2009.

21. Meurant G. Brain Mechanisms for the integration of posture and movement. Amsterdam: Elsevier Science Publishing; 2003.

22. Winkelstein BA. Orthopaedic Biomechanics. CRC Press, New York: Taylor \& Francis Publishing; 2013.

23. Walsh WR. Repair and regeneration of ligaments, tendons, and joint capsule. New York: Human Press; 2007.

24. Merriman LM, Tollafield DR. Assessment of the Lower Limb. Philadelphia: Lippincott Williams and Wilkins; 1995.

25. Salvo SG. Mosby's pathology for massage therapists. 3rd ed. Atlanta: Mosby Elsevier; 2008.
26. Wong DA, Transfeldt E. Macnab's backache. Philadelphia, PA: Lippincott Williams and Wilkins; 2006.

27. Acton A. Somatosensory disorders-advances in research and treatment. Atlanta: Scholarly Editions; 2012.

28. Bradley WG, Daroff RB, Fenichel GM, et al. Neurology in clinical practice: principles of diagnosis and management. Atlanta: Elsevier Science Publishing; 2004.

29. Russell SM. Examination of peripheral nerve injuries: an anatomical approach. New York, USA: Thieme Medical Publishing; 2011.

30. Oh SJ. Clinical electromyography: nerve conduction studies. Philadelphia: Lippincott Williams and Wilkins; 2003.

31. Pal P. Textbook of practical physiology. Orient Longman, Andhra Pradesh; 2005.

32. Iansek R, Morris ME. Rehabilitation in movement disorders. Cambridge: Cambridge University Press; 2013.

33. Suchowersky O, Comella C. Hyperkinetic Movement Disorders. New York: Springer Publishing; 2012.

34. Biller J. Practical neurology. Philadelphia: Lippincott Williams and Wilkins; 2012.

35. Manning WH. Clinical decision making in fluency disorders. Washington DC: Library of Congress Publishing; 2009.

36. Skinner S. Understanding clinical investigations: a quick reference manual. Atlanta GA: Elsevier Science Publishing; 2005.

37. Canobbio MM. Mosby's Handbook of patient teaching. 3rd ed. Atlanta: Mosby Elsevier; 2006.

38. Bastable SB. Essentials of patient education. Washington DC: Jones \& Bartlett learning; 2006.

39. Lorig K. Patient education: a practical approach. Washington, DC: Library of Congress Publishing; 2001.

40. Redman BK. The practice of patient education: a case study approach. 10th ed. Philadelphia: Lippincott Williams and Wilkins; 2007. 